\title{
A static position-adjustment method for the motion prediction of the Flexible Floating Collision-Prevention System ${ }^{1}$
}

\author{
Xu-jun Chen ${ }^{\mathrm{a}, \mathrm{b}, \mathrm{c}, *}$, Wei Yu ${ }^{\mathrm{a}}$, Guang-huai Wu ${ }^{\mathrm{a}, \mathrm{d}}$, Pandeli Temarel ${ }^{\mathrm{b}}$, Jun-yi Liu ${ }^{\mathrm{a}, \mathrm{b}, \mathrm{c}}$
}

a) College of Field Engineering, PLA University of Science and Technology, Nanjing 210007, China

b) Fluid Structure Interactions Group, University of Southampton, SO16 7QF, United Kingdom

c) China Ship Scientific Research Center, Wuxi 214082, China

d) Nanjing Guangbo Engineering Technology co., Ltd, Nanjing 210007, China

\begin{abstract}
The Flexible Floating Collision-Prevention System (FFCPS), used to prevent collision between uncontrolled ships and non-navigational bridges, comprises cable chains, floating structures and mooring system. Its main working principle is to use the sliding of the mooring systems in the role of energy dissipation. It can convert the kinetic energy of the ship into internal energy, and thus achieve the effect of avoiding a ship collision. The force relationships between various components of the system and ensuing simplified numerical models are firstly established in order to study the movement of the FFCPS. Subsequently, using suitable assumptions, the method of position adjustment to approach equilibrium condition is introduced. This method is based on the concept of statically determinate equilibrium in each step. The feasibility of the method proposed in this paper is verified by comparing the calculated results with model test measurements and published reference predictions. From these comparisons it is concluded that this method can be used in the preliminary design stage of the FFCPS.
\end{abstract}

Keywords: offshore structure, ship-bridge collision avoidance, block force, mooring system, FFCPS

\section{Introduction}

As accidents involving ship collisions occur more frequently, more and more scholars and researchers are involved with the ship-bridge collision problems. There have been many studies focusing on collision risk assessment [1-5]. The application of Integrated Bridge Systems incorporated on a ship and the corresponding new methods for determining safe ship trajectory [6-8] increase the safety of navigation.

1 The project was supported by the National Natural Science Foundation of China (Grant No. 51679250;51379213), The National Key Technology R\&D Program(Grant No. 2014BAB16B05) and High-tech Ship Research Projects Sponsored by Ministry of Industry and Information Technology(Grant No. 2016-22).

* Corresponding author. Tel: +86-25-80821315, E-mail: chenxujun213@ sina.com 
Nevertheless, these solutions cannot thoroughly prevent the ship-bridge collision, hence, the design of ship-bridge collision avoidance system have been paid more attentions [8,9]. In these studies, simulation based on finite element analysis is one of the most frequently used methods [10-13]. .

There are many new types of anti-collision devices for protecting bridges. Lei et al.[14] analyzed various aspects of the bridge-collision problem, putting forward the key principles of the "initiative anti-collision" concept and presenting a corresponding simplified model. Zhu et al.[15] proposed a simplified energy-based analysis method to estimate the lateral deflection of the flexible pile-supported protective structures that are subjected to a given impact energy. Chang et al [16] analyzed buoys and their anti-collision features, and provided several theoretical suggestions for the design of anti-collision buoys. $\mathrm{Wu}$ et al.[17] designed an "energy consuming collision-prevention system of long distance anchor moving", which is installed at a suitable distance away from the bridge to prevent the collision between ship and non-navigational bridge. This system, namely the Flexible Floating Collision-Prevention System (FFCPS), is made up of buoys, connection cables and mooring system, as shown in Figure 1. Chen et al.[18-20] studied the methods for simulating the motion of the system when collided with uncontrolled ships. The kinetic energy and potential energy of the moored collision-prevention system, when collided with a ship, were calculated through the kinetic energy theorem and the mooring force equation. Then the approximately static equations are solved by a numerical iterative calculation method for the FFCPS. Xie and $\mathrm{Li}$ [21] proposed a system made up of independent anti-collision piers, interception rope chains, steel floating bodies and anchor ingot sink block. It is similar to FFCPS and it has been applied in the Hangzhou Bay Sea-Crossing Bridge of China. Based on position adjustment to approach equilibrium condition, a new method for the motion analysis of the FFCPS is presented in this paper. The numerical model based on this method is similar to the model proposed in reference [20], but there are some differences between them. In reference [20], the position of each component is adjusted simultaneously in each micro-time step, while the elements are adjusted locally during each micro-displacement in this essay. By comparing the numerical results obtained from the proposed method with those from published numerical and experimental results, its feasibility for application in the preliminary design process is verified. Furthermore, the proposed model can obtain faster convergence rate.

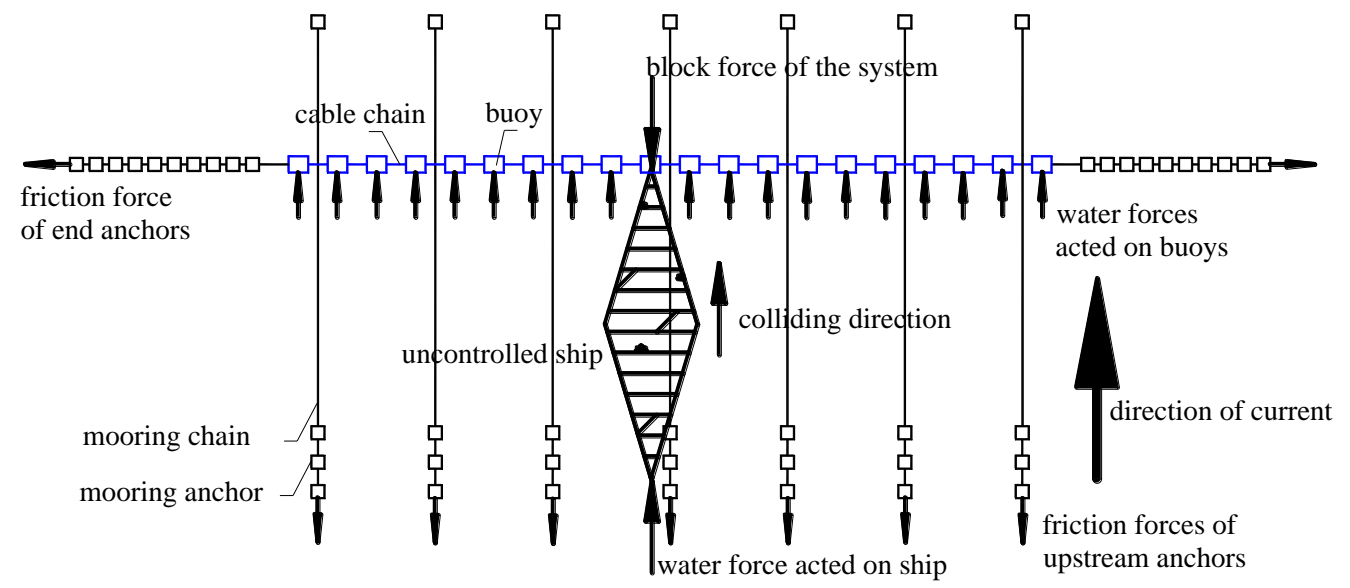

Figure 1 Schematic diagram of Flexible Floating Collision-Prevention System 
FFCPS comprises the water surface blocking system and mooring system. The former consists of buoys and the connecting cable chains, which are floating on the water surface to prevent the colliding ship. The mooring system is composed of mooring chains and anchors. Before collision, except for the internal loads of connecting cables and mooring lines etc., the water forces acting on the buoys and the friction forces of anchors shown in Figure 1 are the external forces acting on FFCPS. When a ship collides with the system, the block force of the system acting on the ship has the same value (but opposite direction) with the push force that the ship exerts on the system. At the same time, the water force related to the relative velocity between the ship and current is another external force acting on the ship, as shown in Figure 1.

Figure 2 illustrates how the FFCPS functions in a model test. In most cases, the current is generated and causes the ship to drift. When the uncontrolled ship collides with the system, firstly the water surface components of the system will move with the ship. When the horizontal force component of a mooring chain reaches the maximum friction force between the anchor and the sea bed, the anchor will slide on the sea bed under the action of increased mooring force induced by the collision. The mooring system, through the sliding of the anchors, absorbs the energy of the colliding ship, including the initial kinetic energy and the work obtained from the current in the later process. It should be stated that a small part of the kinetic energy and work might also be transformed into the potential energy in the mooring system, kinematic energy of the buoys and cable chains and strain energy of the structure, but they are not be considered in this paper. If the summation of the friction forces of the anchors, namely the maximum block force, is bigger than the total current force (as well as wind and wave force, if relevant, but not considered in this paper) acting on the ship and buoys, the ship will be controlled under the block force of the FFCPS in the end. The maximum block force and the duration of the collision are controlled by adjusting the weight and number of gravity anchors. In addition, the block force of FFCPS increases gradually during the collision with the ship movement on the whole, which contributes to reducing the damage on the ship. FFCPS can automatically adapt to changes of water level and it can be used repeatedly to block collision with large-tonnage ships in different hydrological and meteorological conditions.

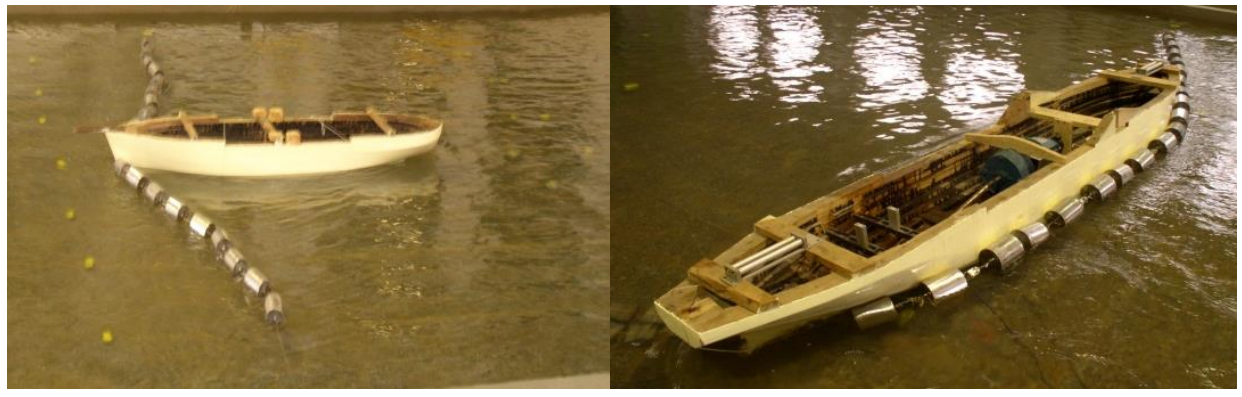

Figure 2 Model test of FFCPS

\section{Loads and static analysis of FFCPS}

The mechanical analysis is the basis for analyzing the FFCPS. The FFCPS is divided into several elements, namely the colliding ship, the connecting cable chains, the buoys and the mooring system. The internal forces of each element have their own characteristics. Furthermore, there are various external 
forces acting on the FFCPS. Each part of the FFCPS can be analyzed in an approximately static condition as no dynamic loads are considered in this paper.

\subsection{Mechanical Analysis of the Colliding Ship.}

There will be energy losses as a consequence of the ship collision. Part of the kinetic energy is converted into deformation energy, representing the energy losses due to the deformation of the ship and the buoys. Their estimation methods should be based on material properties, as well as the internal structure of the ship and the buoys. Besides, the rigidity and mass of the ship are much greater than those of the buoy-chain system. Hence, we do not study the collision at initial instant and the deformation of the ship, but focus on studying the movement of the FFCPS during the collision process in this paper. Compared with the traditional collision, such as the ship colliding directly with piers, walls and ground [22] etc., the duration of the collision for our case is much longer and the acceleration will be very small, therefore the added mass relevant to the acceleration against the forward speed of the ship could be omitted in the simulation. That is to say, no dynamic effect has been considered. Accordingly, the added mass and hydrodynamic damping are not considered in this paper. The focus is on the large deformation of the collision prevention system with buoys connected by lines. As a consequence of these assumptions, the kinetic energy of the ship is unchanged at the beginning of the collision and the ship will continue to move with its pre-collision speed. The pre-collision speed will be equal to flow velocity in many cases as the colliding ship is an uncontrolled ship. That is to say the speed is small and the collision process can be approximated as steady state. Since the speed variation is small in each step, the added mass and hydrodynamic damping are also small and can be omitted. If 'the ship continues to move with its pre-collision speed', the deformation of the mooring system will become larger and larger. As a result, the block force acting on the ship will be increasingly large, and the speed of the ship will become smaller and smaller under the action of the block force. Final equilibrium with zero ship speed is reached on the condition of the summation of the friction forces of the anchors, namely the maximum block force, is bigger than the total current force acting on the ship and all the buoys.

During the collision process, there are several forces acting on the ship, namely the forces induced by the reaction of the FFCPS and the flow force. The flow force calculation depends on the heading of the ship and the direction of flow. According to the JTS 144-1-2010 standard [23], the flow force components are as follows:

$$
\begin{aligned}
& F_{y c}=C_{y c} \frac{\rho}{2} V^{2} S \\
& F_{x c}=C_{x c} \frac{\rho}{2} V^{2} A_{y c}
\end{aligned}
$$

$F_{y c}$ in Equation (1) is the longitudinal component of the flow force, and its direction is along with the longitudinal direction of ship; $F_{x c}$ in Equation (2) is the lateral component of the flow force, which has the same direction with the ship lateral direction. $C_{y c}$ and $C_{x c}$ are the corresponding force coefficients; 
$\rho, V, S$ and $A_{y c}$ denote the density of water, the speed of the ship relative to the water, the surface area of the ship below the waterline and the underwater projected area of the ship perpendicular to the relative current direction, respectively.

The longitudinal force coefficient is as below:

$$
C_{y c}=0.046 R_{\mathrm{e}}^{-0.134}+b
$$

where $R_{\mathrm{e}}$ is the Reynolds number and it is related to the flow velocity, the length of the ship and the kinematic viscosity coefficient of water. According to the specification, when the water temperature is $20^{\circ} \mathrm{C}$, the value of $R_{\mathrm{e}}$ is $1.00 \times 10^{-4} \mathrm{~m}^{2} / \mathrm{s}$. $b$ in Equation (3) is a coefficient related to the block coefficient of the ship, the ratio of beam to draught and the angle between the longitudinal axis of the ship and the water flow. In this paper the block coefficient of the model ship is 0.625 , the ratio of beam to draught is 2.2 , and the angle between the longitudinal axis of the ship and the water flow is less than $15^{\circ}$. In such conditions, the value of $b$ could be set as $0.0[23]$.

The surface area of the ship below the waterline can be calculated as follows [23]:

$$
S=1.7 L D+C_{b} L B
$$

where $L, D, C_{b}$ and $B$ are the length, draught, block coefficient and breadth of the ship respectively.

The lateral force coefficient can be expressed as below:

$$
C_{x c}=a_{1} \frac{\pi \theta}{180}+b_{1}
$$

The relative depth is the ratio of the water depth in the working condition and the ship draught. In accordance with the specification of the standard [23], when the value of the relative depth is 1.1, the parameters in Equation (5) are listed as follows: $\theta=90^{\circ}, a_{1}=1.70, b_{1}=0.31$. The underwater projected area of the ship perpendicular to the relative current direction is as below:

$$
A_{y c}=B^{\prime} \sin \theta=B^{\prime}=L D
$$

where $B^{\prime}$ denotes the transversely projected area of the underwater part of the ship. 


\subsection{Static Mechanical Analysis of the connecting cable chain.}

The connecting cable chain plays an important role in the function of the FFCPS by connecting the floating structures mainly comprising buoys. When calculating the tension of the block chain, the weight of the connecting cable chain must be considered and the solution to the catenary must be used. The connecting cable chain is a type of flexible connection, which can only result in tensile stress. Due to its weight, the shape of connecting cable and its forces satisfy the corresponding catenary equations. Therefore, in addition to the horizontal tensile force, there is a vertical force which is equal to the value of its weight at both ends. The force diagram of the joints of one part of the connecting cable chain is shown in Figure 3.

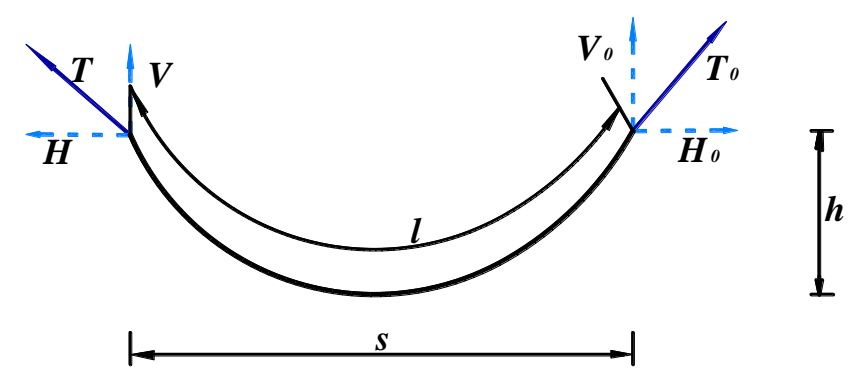

Figure 3 The force diagram of one part of the connecting cable chain

$T_{0}$ and $T$ are the pulling forces of the connecting cable at two ends; $H_{0}$ and $V_{0}$ are the horizontal and vertical components of $T_{0}$, while $H$ and $V$ are the horizontal and vertical components of $T$. In Figure $3, h$ is the vertical projection of connecting cable chain. $l$ is the length of one part of the connecting cable chain and $s$ denotes its horizontal projection. According to the catenary equation, the relationship between the variables can be easily expressed as follows[24]:

$$
\begin{aligned}
& H=-H_{0} \\
& V_{0}+V=w l \\
& T=\sqrt{H^{2}+V^{2}} \\
& T_{0}=\sqrt{H_{0}^{2}+V_{0}^{2}} \\
& s=-H_{0}\left(\frac{l}{E A}+\frac{1}{w} \ln \frac{V+T}{T_{0}-V_{0}}\right)
\end{aligned}
$$




$$
h=\frac{1}{2 E A w}\left(T^{2}-T_{0}^{2}\right)+\frac{1}{w}\left(T-T_{0}\right)
$$

In these equations $w, E$ and $A$ denote the weight per unit length, equivalent Young's modulus and cross-section area of the cable chain, respectively.

There will be a certain pre-tension in the system prior to the collision, keeping the FFCPS at a stable state. That is to say, the tensile stresses of the cable chains keep the FFCPS relatively stationary under the action of water and other external forces. The values of the pre-tension directly determine the shape of the catenary chain. It cannot be too small, which implies that the vertical projection of the connecting cable chain is quite small compared with half of the horizontal projection of the connecting cable, namely $h<<\frac{s}{2}$. Hence, according to the geometric relationship depicted in Figure 4, it can be seen that:

$$
H=\frac{V s}{2 h}
$$

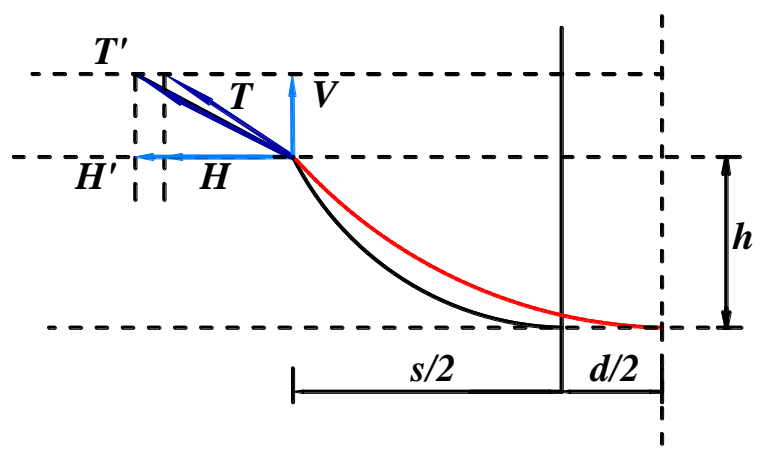

Figure 4 Diagram showing the changes of cable chain tension

The horizontal component of the tension of the connecting cable chain changes with the variations of the external forces. This change is directly caused by the position alternations of the two ends of the connecting cable chain. If the variation of the horizontal projection of the connecting cable chain is $d$ and the vertical projection of the connecting cable remains basically unchanged, the variation of tension in the horizontal direction $\Delta H$ can be expressed as below:

$$
\Delta H=\frac{V}{4 h} d
$$


Equation (14) indicates that, in the case of pre-loading the cable chain with a large tension, the variation of the tension horizontal component is proportional to the change of horizontal projection of the connecting cable chain. This is similar to a linear elastic model of cable. During the process of collision, the connecting cable chain is always in tension, hence, there is no need to calculate the tension through the catenary method. A linear elastic cable model is sufficient for obtaining an accurate prediction. The weight of the connecting cable chain can be ignored, simplifying the calculations.

\subsection{The force equilibrium of the buoys}

Buoys in the FFCPS enable the connecting cable chains and themselves to float on the water surface to prevent the ship from colliding with the bridge. The internal forces in the system arise from the connecting cable chain. The tensions pull the buoys on the ends of the cable chains. The main external forces are the water force and the collision force. The buoys are in equilibrium under the internal and external forces. In the process of collision, the buoys floating status may change, i.e. they will experience heaving motion. However, this motion is assumed to be negligible compared with the deformation of the system. In this case, the gravity and buoyancy forces of the buoys are always in equilibrium, hence, the forces on the buoys can be simplified in a two-dimensional plane, ignoring the effect of gravity and buoyancy. The coordinate system $o x y$ in Fig. 5 denotes the global coordinate system of the FFCPS. $O_{i}$ is the (motion) centre point of the buoy; $F_{\mathrm{W} i}$ is the external force on the buoy; $\alpha$ is the angle subtended between axis $o x$ and the direction of $F_{\mathrm{W} i} ; M_{\mathrm{W} i}$ is the torque of the buoys induced by the external force. The angle $\alpha$ considered here can simulate the geometrical nonlinearity of the buoys. $F_{\mathrm{W}_{i}}$ and $M_{\mathrm{W} i}$ are mainly induced by current in this paper, as the wind and wave loads are not included in the analysis. $F_{\mathrm{A} i}$ and $F_{\mathrm{B} i}$ are the horizontal component of the cable force acting on the left and right end of buoy respectively. $\alpha_{A i}$ is the angle subtended between $F_{\mathrm{A} i}$ and $x$-axis. $\alpha_{\mathrm{B} i}$ is the angle subtended between $F_{\mathrm{B} i}$ and the axis of $o x$, respectively. Accordingly, the forces on the buoy can be evaluated as follows:

$$
\left\{\begin{array}{l}
\sum F_{x}=F_{\mathrm{W} i} \cos \alpha+F_{\mathrm{A} i} \cos \alpha_{\mathrm{A} i}+F_{\mathrm{B} i} \cos \alpha_{\mathrm{B} i} \\
\sum F_{y}=F_{\mathrm{W} i} \sin \alpha+F_{\mathrm{A} i} \sin \alpha_{\mathrm{A} i}+F_{\mathrm{B} i} \sin \alpha_{\mathrm{B} i} \\
\sum M=M_{\mathrm{W} i}+\left(F_{\mathrm{B} i} \cos \alpha_{\mathrm{B} i}-F_{\mathrm{A} i} \cos \alpha_{\mathrm{A} i}\right)\left(y_{\mathrm{B} i}-y_{\mathrm{A} i}\right) / 2 \\
+\left(F_{\mathrm{A} i} \sin \alpha_{\mathrm{A} i}-F_{\mathrm{B} i} \sin \alpha_{\mathrm{B} i}\right)\left(x_{\mathrm{B} i}-x_{\mathrm{B} i}\right) / 2
\end{array} .\right.
$$




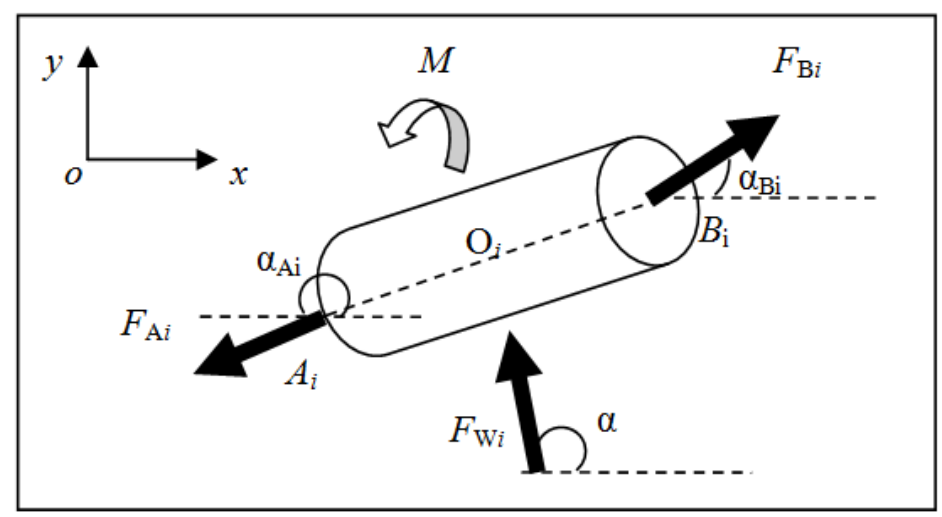

Figure 5 Schematic diagram of two-dimensional forces on the buoy

In the working state of the FFCPS, the torque $M_{\mathrm{w} i}$ is mainly induced by the water. Since the flow forces acting on the buoys are approximately symmetric about the motion centers of the buoys, the torques caused by water can be neglected in the calculation process. An exception to this assumption is for the buoy in the impact position.

\subsection{Mechanical Analysis of the mooring system}

The mooring system is a key component of the FFCPS, not only playing the role of locating the system and but also absorbing the impact energy of the system by the sliding movement of the anchors. The movable anchors ensures that the mooring chain and connecting cable chain will not be broken.

The friction between the anchors and the seabed cannot be too large, otherwise, the connecting cable chain or the mooring chain will break. At the same time, it also should not be too small, because in this case the FFCPS cannot be located in the designed position under the current forces, and it cannot provide sufficient block force to prevent the collision ship. Therefore, the best type of anchor is the gravity anchor as it is easier to slide on the seabed and can provide a relatively large friction in the sliding process. The number of gravity anchors and their weights are the most important parameters to be studied by the designers and researchers.

The force of the mooring system, prior to the collision, can be calculated by solving the catenary equation. In this case the horizontal components of mooring forces are considered whilst their vertical components are ignored. The same process can be used after the collision with the ship, albeit for a short time step. During this time step, the horizontal component of a mooring force is less than the sliding friction between the anchor and the seabed. In the process of the anchor moving, the horizontal component of the mooring force will be equal to the sliding friction between the anchor and the seabed since the current loads acting on the mooring lines and anchors are neglected in this paper. The horizontal component of the mooring force will appear to fluctuate within a certain range due to the asymmetric geology of the seabed as shown in Figure 6(a). The case of constant horizontal force, shown in Figure 6(b), was used in the simulation of this paper as there are no test values on the sea bed. 


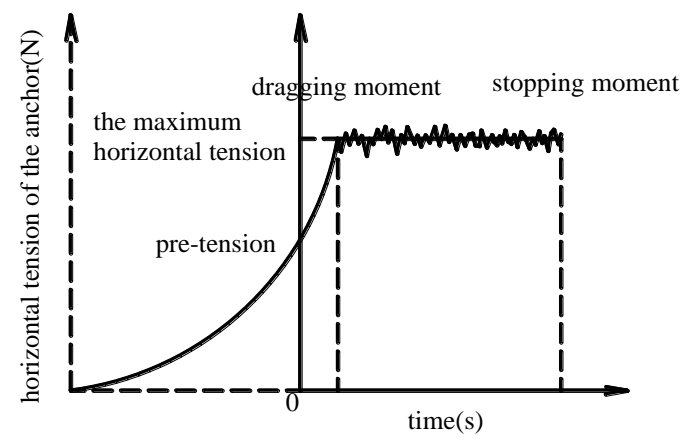

(a)

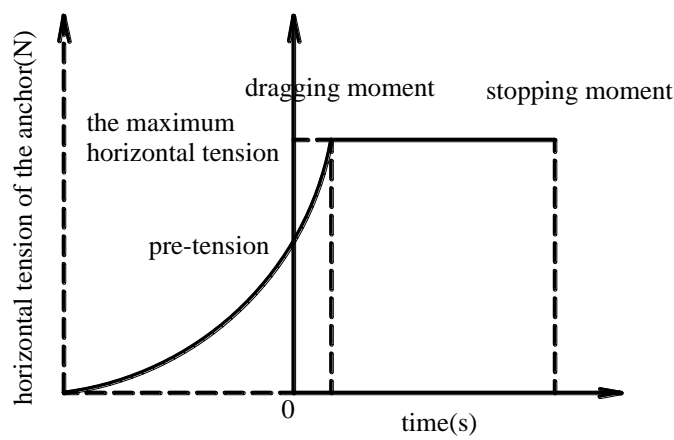

(b)

Figure 6 Time history plot of the horizontal component of the mooring force

\section{Description of the Solution Method}

As the components of the FFCPS are connected by connecting cable chains, the relationships between the movements of different elements of the system are complicated. The movement of one element will influence that of the others. Here, a static analysis method is used, as in the process of collision each component tends to move to an equilibrium position. In other words, each component will move in the direction of the resultant force at each instant. Therefore, if the process is divided into many, small, time steps, the resultant force of each component will be very small at the end of each time step. The FFSCS stops at a relative equilibrium state. In order to analyze the process of collision, the whole process is analyzed step by step in accordance with the position variation of the impact point. The colliding ship will move forward with the impact point. The distance traveled can be divided into a lot of micro displacements. The ship velocity is assumed to be constant in each micro displacement, but it varies in different steps. In each step of the process, the movement of the colliding ship and the impacting part of the FFCPS will cause the movement of other elements. All elements interact with each other; nevertheless, the greatest effect on the movement of one element is due to the movements of the nearest elements. The process of iterative calculation is shown in Figure 7.

Firstly a micro displacement is set at the impact point, and its direction is the same as the direction of the ship velocity. Then, the positions of the remaining elements from the near to the distant relative to the impact point are gradually adjusted. When the position of one element is adjusted, only its adjacent elements are taken into consideration. This results in a new position for each element. The iterative process results in the resultant force being smaller than before. The positions are adjusted step by step until all of the resultant forces, except that of the impact point, are small enough. At that time, the micro displacement step is completed and the time step elapses, hence the velocity of each element at the end of the time step can be calculated using Newton's law. If the energy of the colliding ship is less than a predetermined small value, the collision process is stopped, otherwise the next step of the micro displacement is entered and the process continues. 


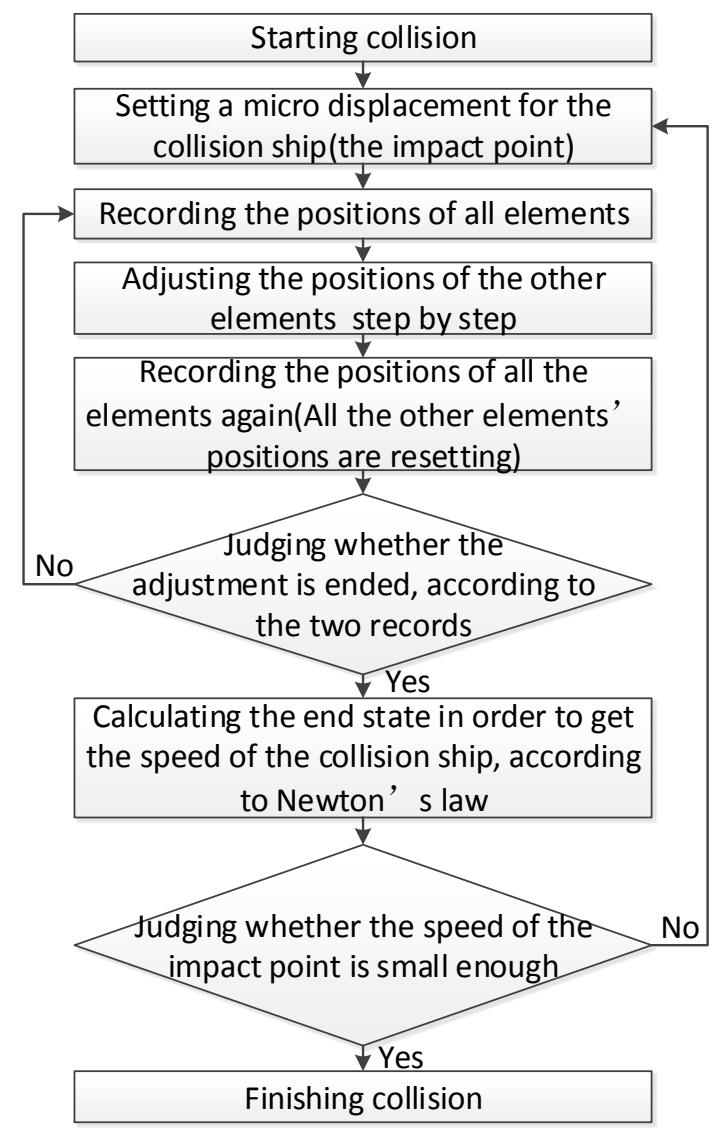

Figure 7 Flow diagram of the iterative process

In addition to all buoys, all of the intersections of the connecting cable chains should be treated as elements. The intersection points of all the elements on the water surface and the mooring system under water are shown in Figure 8.

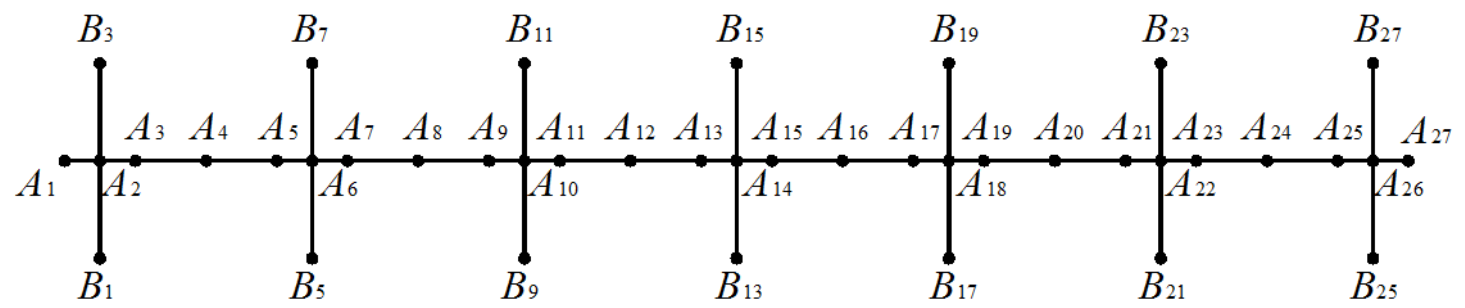

Figure 8 Diagram of the elements and their intersections

In each calculation step, the positions of all the buoys should be adjusted to achieve the equilibrium of the FFCPS in that step. The points in the system are marked as $A_{1}, A_{2}, A_{3}, \ldots, A_{n}$ respectively, as shown in Figure 8 , where $n$ is the amount of the elements. The collision point moves first in the process of adjusting the attitude of the FFCPS, and its coordinate is $\left[A_{i}\right]$ (or they can be expressed as a coordinate set $\left\{\left[A_{i}\right],\left[A_{i+1}\right], \ldots,\left[A_{j}\right]\right\}$ under the condition of transverse collision). Other marked points will move with 
the collision point. A micro displacement $\Delta d$ to the collision point is given. In this step, the collision point is fixed in its position. Based on the method of position adjustment to approach equilibrium condition, the positions of the marked points are adjusted from the nearest to furthest step by step. Adjustment functions $f_{\mathrm{F}}$ for forehead collision and $f_{\mathrm{T}}$ transverse impact are defined as follows.

$$
\begin{aligned}
& {\left[A_{k}^{\prime}\right]=f_{\mathrm{F}}\left(\left[A_{k-1}\right],\left[A_{k+1}\right], X_{k}, Y_{k},\left[A_{k}\right]\right)} \\
& \left\{\left[A_{k}^{\prime}\right],\left[B_{k-1}^{\prime}\right],\left[B_{k+1}^{\prime}\right]\right\}=f_{\mathrm{T}}\left(\left[A_{k-1}\right],\left[A_{k+1}\right],\left[B_{k-1}\right],\left[B_{k+1}\right],\left[A_{k}\right]\right)
\end{aligned}
$$

where $\left[A_{k}\right]$ is the coordinate of marked point $A_{k},\left[A_{k-1}\right] \operatorname{and}\left[A_{k+1}\right]$ are coordinates of the marked points next to $A_{k},\left[B_{k-1}\right]$ and $\left[B_{k+1}\right]$ are, respectively, the position coordinates of upstream and downstream mooring points relevant to the intersection marked as $A_{k} . X_{k}$ and $Y_{k}$ are, respectively, the horizontal and vertical components of the resultant force of marked point $A_{k} .\left[A^{\prime}{ }_{k}\right]$ and $\left[B^{\prime}{ }_{k}\right]$ are the adjusted position coordinates of marked points $A_{k}$ and $B_{k}$. These are illustrated in Figure 9, where the marked point $A_{13}$ is the ship collision point. Equation (16) is used to adjust the position of marked point $A_{12} . A_{11}$ and $A_{13}$ are the marked points next to $A_{12}$.

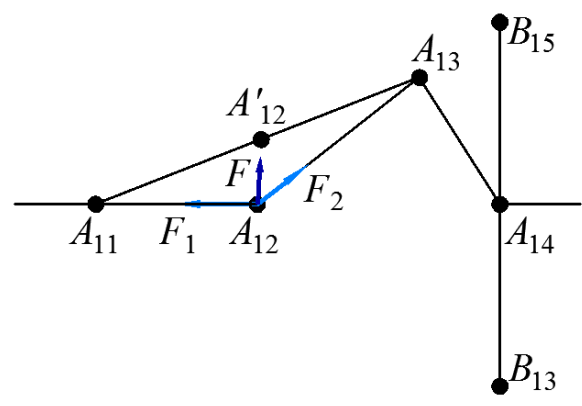

Figure 9 Schematic diagram of buoy position adjustment

The value of the tension $F_{1}$ between $A_{12}$ and $A_{11}$ together with the tension $F_{2}$ between $A_{12}$ and $A_{13}$, can be obtained according to the case of the elongation of the chain as shown in Figure 10. The resultant force $F$ acted on the marked point $A_{12}$ can be calculated by adding the external forces $X_{12}$ and $Y_{12}$. According to the principle of force equilibrium, the marked point $A_{12}$ should move a corresponding distance 
in the direction of resultant force. Thus, the value of the resultant force $F$ is close to zero, and the adjusted marked point becomes $A_{12}^{\prime}$. The adjusted marked point $A_{11}^{\prime}$ can be obtained in the same way.

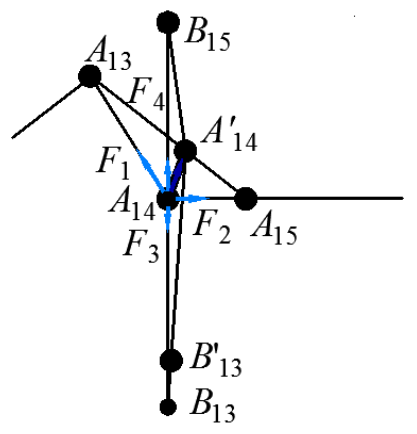

Figure 10 Schematic diagram of intersection position adjustment

Equation (17) can be used to adjust the position of the chain intersection $A_{14} . A_{13}$ and $A_{15}$ are the marked points next to $A_{14} ; B_{13}$ and $B_{15}$ are the upstream and downstream mooring points of the intersection $A_{14}$. When adjusting the position of the marked intersection $A_{14}$, the mooring force between $A_{14}$ and $B_{13}$ as well as the downstream mooring force between $A_{14}$ and $A_{15}$ need to be determined. If one of the mooring forces reaches the value of the friction between the anchor and the seabed, the relevant anchor will move. Then the position of $B_{13}$ or $B_{15}$ will change.

In accordance with this method, the positions of the marked points on both sides of the collision point are gradually adjusted till all the adjustments are completed. Thus a new set of marked point positions $\left\{\left[A_{1}^{\prime}\right],\left[A_{2}^{\prime}\right],\left[A_{3}^{\prime}\right], \ldots,\left[A_{n}^{\prime}\right]\right\}$ is obtained. Then the adjusted distances of each point with the new positions are calculated. If all the horizontal components of the adjusted mooring forces are less than the prescribed value of the friction between the anchor and the seabed, the adjusting process can be stopped and the collision point is allowed to move by another micro displacement $\Delta d$. Otherwise, all the marked points should be adjusted again.

In the process of adjustment, the issue of moving anchors should be considered. When the positions of intersections of the chains are changed, the mooring forces will change correspondingly. When the mooring forces are large enough the anchors will move with the system.

In each step of the adjustment process, the direction of positions of the buoys should be reset to ensure that, the torque of each element is close to zero. If the value of the torque is negative, the buoy should be turned by a small angle clockwise until the torque of the buoy is near to zero. Otherwise, the buoy should be turned anticlockwise. After adjusting the direction of positions of the buoys, the resultant force of the buoys will change. Therefore, the position should be adjusted again to make the resultant force near to zero. 
This process of adjustment is repeated until all of the values are acceptable, ensuring the FFCPS is in equilibrium in this step.

With the gradual movement of the collision point, FFCPS exerts flow forces $F_{x c}$ and $F_{y c}$ on the colliding ship during the process, which gradually increase from zero to their final value as the initial speed of the ship is the same with that of the current. With the movement of anchors absorbing the kinetic energy of the ship, the ship velocity decreases. When the speed of the colliding ship is small enough, such as $0.01 \mathrm{~m} / \mathrm{s}$, the collision process is assumed to have ended, indicating that the FFCPS has stopped the colliding ship.

\section{Numerical Examples}

\subsection{Assumptions and verifications}

The assumptions involved in the numerical simulation of the FFCPS are summarized as follows:

(1) The direction of ship velocity is unchanged and not influenced by external factors in the collision process.

(2) The reaction force exerted on the gravity anchor by the sea bed is equal to the weight of the gravity anchor in water. The sliding friction coefficient of the tank bottom (or seabed) is assumed to be constant, and thus the maximum horizontal tension provided by the mooring system is constant.

(3) The force of the connecting cable chain can be calculated using linear elastic theory.

(4) The force of the water (flow force) is the main external force and the effects of other external forces such as wave and wind forces are not considered, even though they can be important design factors of system.

(5) The colliding ship is driven by the current, the initial speeds of the colliding ship are equal to the different velocities of the current.

During the collision process, the orientation of the ship may change under the action of external forces. A yaw moment might also exist. This may lead to a final position of the ship in which the buoys are always in contact with one side of the ship. For ease of simulation, we selected two cases of ship orientation (i.e. heading and transverse direction respectively). Naturally, the heading direction cases may turn to transverse direction in the end of collision process, but not considered in this paper.

In order to verify the accuracy of the simulation method and corresponding algorithm, the model tests of the FFCPS are used for comparison. Figure 2 illustrates how the FFCPS functions in a model test. The depth of the water is $0.25 \mathrm{~m}$. The buoys are hollow cylinders made of stainless steel. The connection chains and the mooring chains have the same particulars, but have different lengths for different types. The anchors are a single concrete block for the downriver side, three connected concrete blocks for the upriver side and ten connected concrete blocks for the two ends of the system. The details of the test are described by $\mathrm{Wu}$ et al. [17], in which two types of ship models (the gravities of big model and small model are $117.3 \mathrm{~kg}$ and $70.4 \mathrm{~kg}$ respectively) collide with the FFCPS at two different current velocities. In addition the predictions by the current method are compared with predictions by Chen et al. [20]. 
The comparisons between the current predictions and those by Chen et al. [20] are shown in Figure 11, where the Figures (a) and (b) are copy from the reference [20] and the Figures (c) and (d) are the results of this paper. Here, case 1 and case 5 are defined in Table 1 Chen et al. [20]. The selected two cases correspond to the big ship model colliding with FFCPS at a speed of $0.375 \mathrm{~m} / \mathrm{s}$. Here and hereafter, the speeds of the ships are equal to the current velocities in forward heading and transverse movement respectively. Comparing the two sets of predictions, it can be seen that at the end of the collision process, the deformations of the system are similar However, the motion displacements of the anchors (i.e. $\mathbf{M}_{i}$ ) and the colliding ship predicted using the current method are relatively smaller than those of Chen et al. [20]. This implies that the work done by internal frictions, which is converted by the kinetic energy of the ship, is smaller. It can be thought that part of the remainder can be converted to elastic energy in the connecting cable chains, which has not been considered by Chen et al.[20].

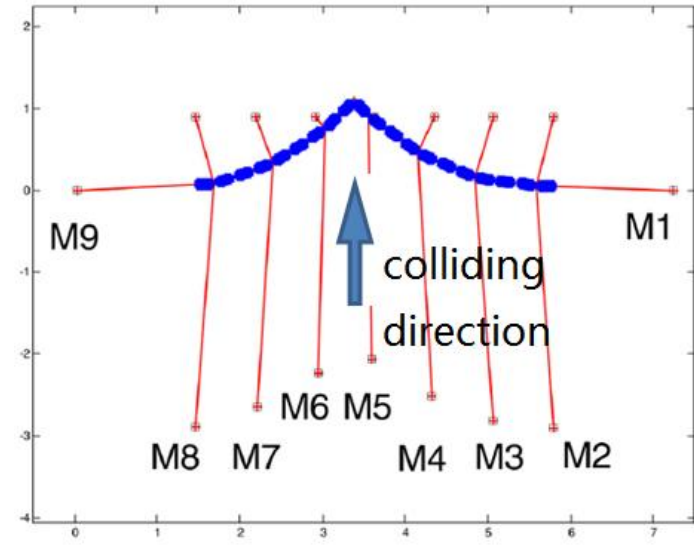

(a) case 1 (big model $\mathrm{V}=0.357 \mathrm{~m} / \mathrm{s}$, reference[20])

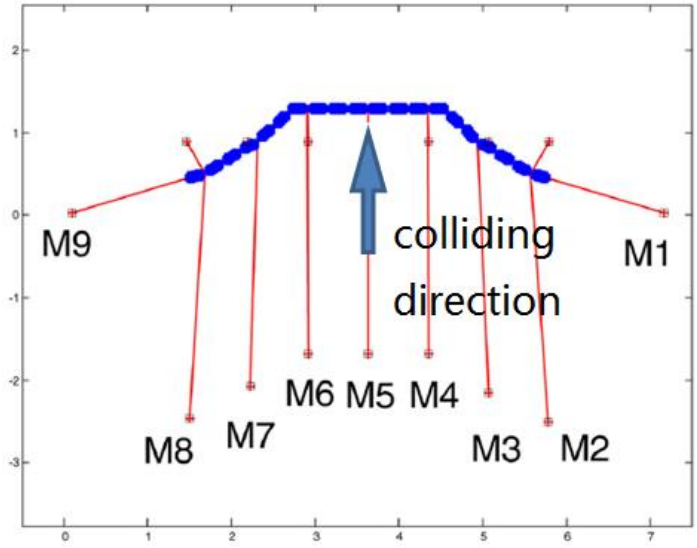

(b) case 5 (big model $\mathrm{V}=0.357 \mathrm{~m} / \mathrm{s}$. reference[20])

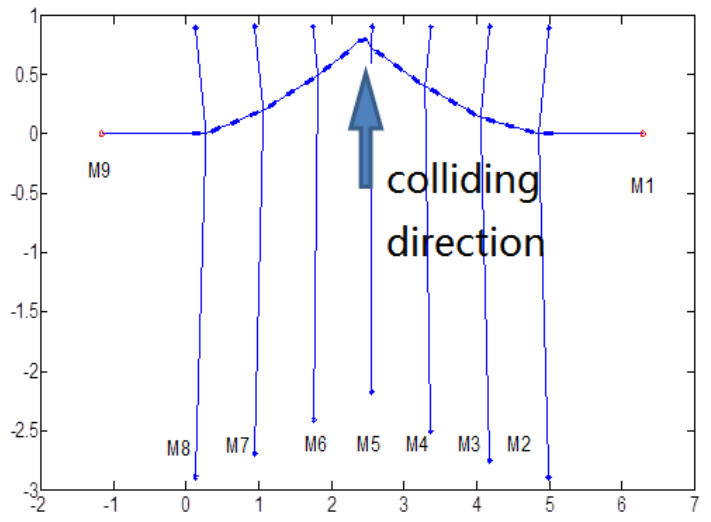

(c) case 1 (big model $\mathrm{V}=0.357 \mathrm{~m} / \mathrm{s}$, this paper)

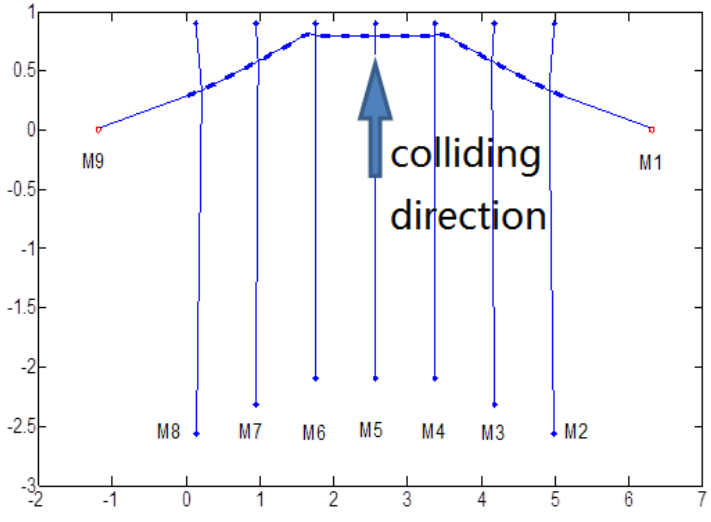

(d) case 5 (big model $\mathrm{V}=0.357 \mathrm{~m} / \mathrm{s}$, this paper)

Figure 11 Deformation of the system for selected cases, comparing with predictions by Chen et al[20] 
Table 1 Ship models and Collision Cases [20]

\begin{tabular}{|c|c|c|c|r|r|r|r|r|}
\hline Cases & 1 & 2 & 3 & 4 & 5 & 6 & 7 & 8 \\
\hline Ship models & Big model & \multicolumn{2}{|c|}{ Small model } & \multicolumn{2}{|c|}{ Big model } & \multicolumn{2}{|c|}{ Small model } \\
\hline Collision cases & $\begin{array}{c}\text { Forehead } \\
\text { collision }\end{array}$ & \multicolumn{2}{|c|}{$\begin{array}{c}\text { Forehead } \\
\text { collision }\end{array}$} & \multicolumn{2}{|c|}{$\begin{array}{c}\text { Transverse } \\
\text { collision }\end{array}$} & \multicolumn{2}{|c|}{$\begin{array}{c}\text { Transverse } \\
\text { collision }\end{array}$} \\
\hline Velocities $(\mathrm{m} / \mathrm{s})$ & 0.357 & 0.254 & 0.357 & 0.254 & 0.357 & 0.254 & 0.357 & 0.254 \\
\hline
\end{tabular}

The anchor displacements in a forward heading collision are shown in Figure 12, compared with test values [17] and literature values [20]. They are similar to each other. Through calculations for a variety of work cases, it is concluded that if the ship collides at the same position with the FFCPS, the order of the values of the anchor dragging distances is certain, namely, excluding the end-anchors M1 and M9, the order of the values of the anchor dragging distances is M5, M6, M4, M7 , M3, M8 and M2, irrespective of type and speed of the colliding ship.
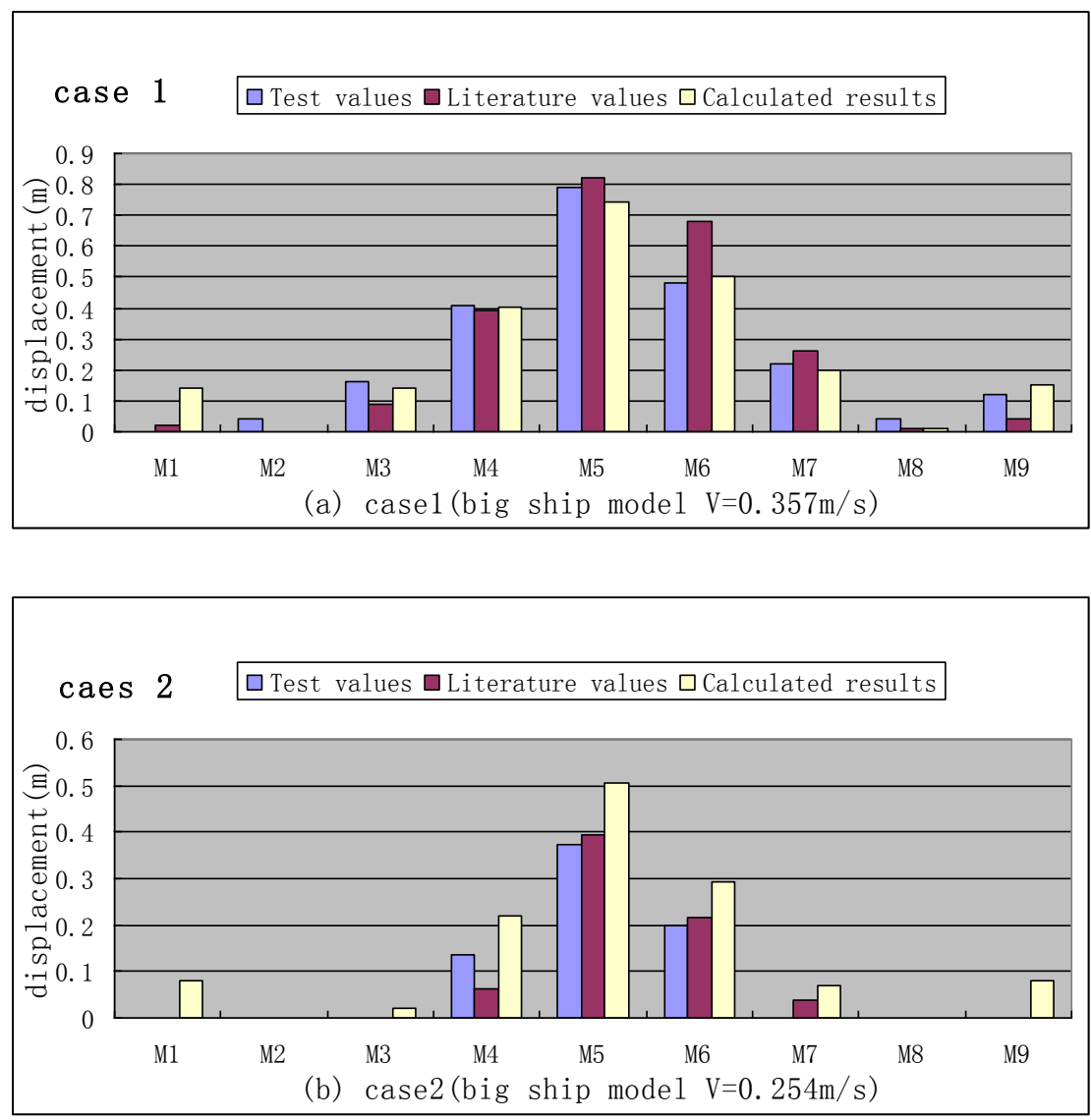

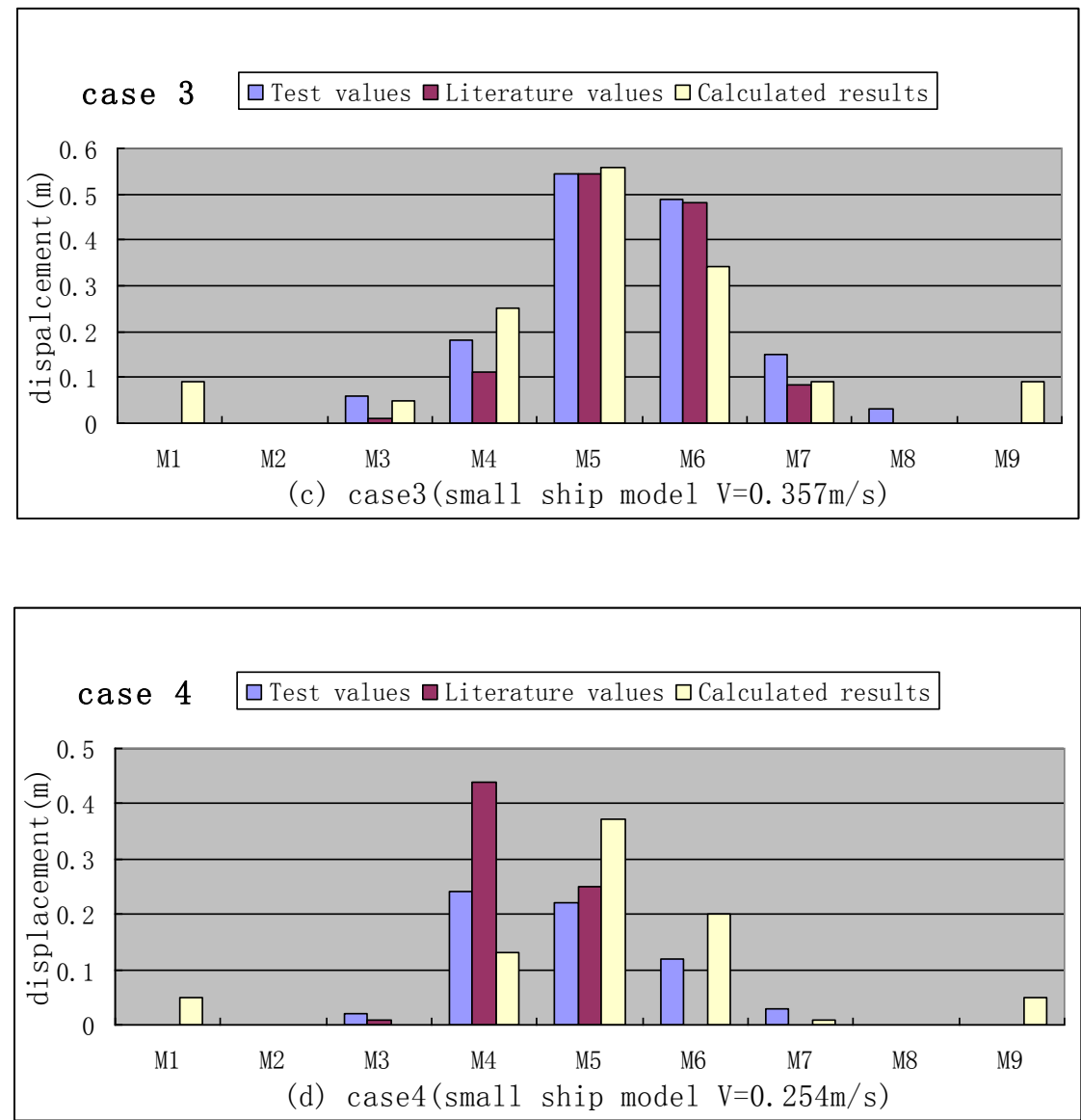

Figure 12 Comparisons of anchor displacements in a forward heading collision

Differences can also be observed in Figure 12. For example, the end-anchors M1 and M9 have moved by varying distances in the results of this paper, whilst those of the model results and published work are much smaller. This is because of the linear elastic model used in the calculation of the chain forces, and the elastic modulus is set to a big value of $3 \times 10^{11} \mathrm{~Pa}$ [25]. This implies that the connecting cable chain is hard to be extended. In addition, the connecting cable chain is tighten in the simulation but could be a little loose in the test, hence there is a small displacement value of end anchors in the simulation but 0 in the test.

\subsection{Analysis of the block forces}

The block force provided by the FFCPS in the process of ship collision is analyzed next, as illustrated in Figure 13. The magnitude of the block force is directly related to two factors, namely the values of pulling forces of the connecting cable chain $\left(T_{1}\right.$ and $\left.T_{2}\right)$ and the angles between the connecting cable chain and the ship's longitudinal axis $\left(\alpha_{1}\right.$ and $\left.\alpha_{2}\right) . \beta_{1}$ and $\beta_{2}$ in Figure 13 are the complementary angles of $\alpha_{1}$ and $\alpha_{2}$ respectively. In the ship collision process, the forces $T_{1}$ and $T_{2}$ gradually go up from the beginning. They will increase to the maximum tensile stress. Obviously, in the beginning of forehead 
collision, the connecting cable is perpendicular to the longitudinal axis of the ship, hence the angles $\alpha_{1}$ and $\alpha_{2}$ will gradually decrease from $90^{\circ}$, while the angles $\beta_{1}$ and $\beta_{2}$ gradually increase from $0^{\circ}$. Based on the force equilibrium conditions, it can be concluded that:

$$
F=T_{1} \cos \alpha_{1}+T_{2} \cos \alpha_{2}
$$

$$
F=T_{1} \sin \beta_{1}+T_{2} \sin \beta_{2}
$$

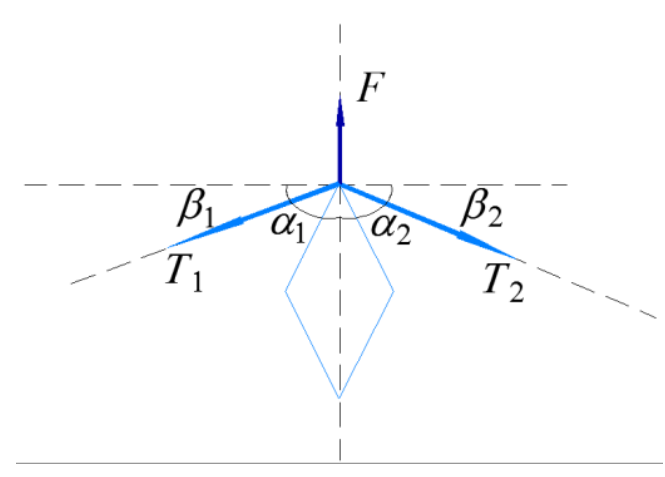

Figure 13 The block force diagram

Finally the block force, i.e. the force exerted by the FFCPS on the ship, was calculated and compared with the results by Chen et al. [20]. These comparisons are shown in Figure 14, as time history of the block forces, where the figures on the left are obtained by the current method and those on the right come from Chen et al. [20]. As can be seen, the orders of magnitudes of both sets of results are the same and there is good correlation between the values of the block forces. The maximum values of the block forces range from $10 \mathrm{~N}$ to $20 \mathrm{~N}$. There are also some differences between the two methods predictions. For example, the results by Chen et al.[20] show that, irrespective of the conditions, the line of block force on the timeline is similar to a linked convex curve. However, the results from the current method are continuous oscillating lines. These oscillations are realistic, since a minor displacement variation may cause one of the anchors to stop or start moving.

In addition, the duration of the collision process by Chen et al.[20] is a little longer than that of the current method. This is related to the variation characteristics in block forces in the two numerical models. If the block force changes slowly, especially at the beginning of the collision, the colliding ship has a high speed for a longer time, resulting in a longer collision process. Comparing the different conditions studied in this paper, it can be concluded that: if the flow rate is greater, the time of the collision will be longer; if the colliding ship is bigger, the duration time of the collision will also be longer. This is consistent with the objective law. 

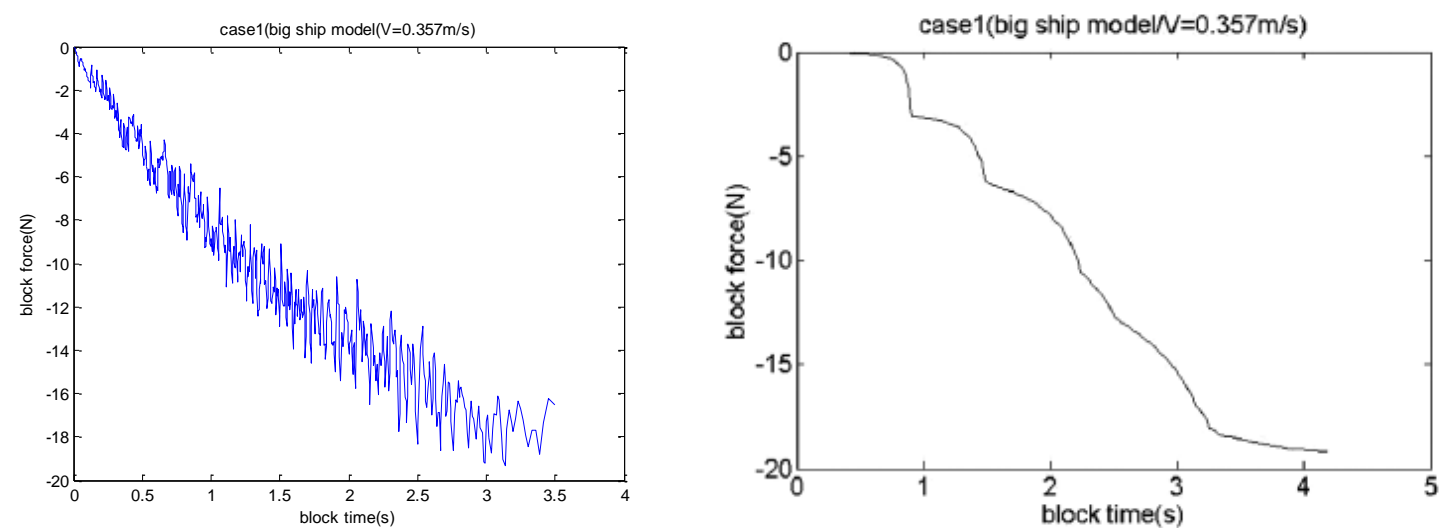

(a) case 1（big model $\mathrm{V}=0.357 \mathrm{~m} / \mathrm{s}$ )
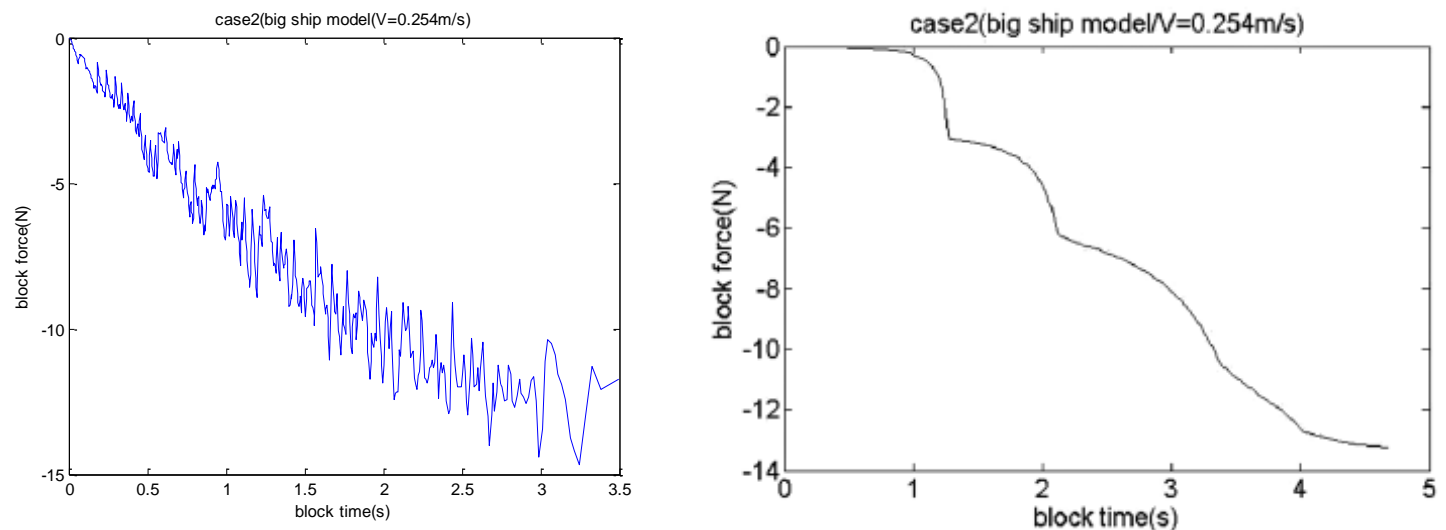

(b) case 2 (big model $\mathrm{V}=0.254 \mathrm{~m} / \mathrm{s}$ )
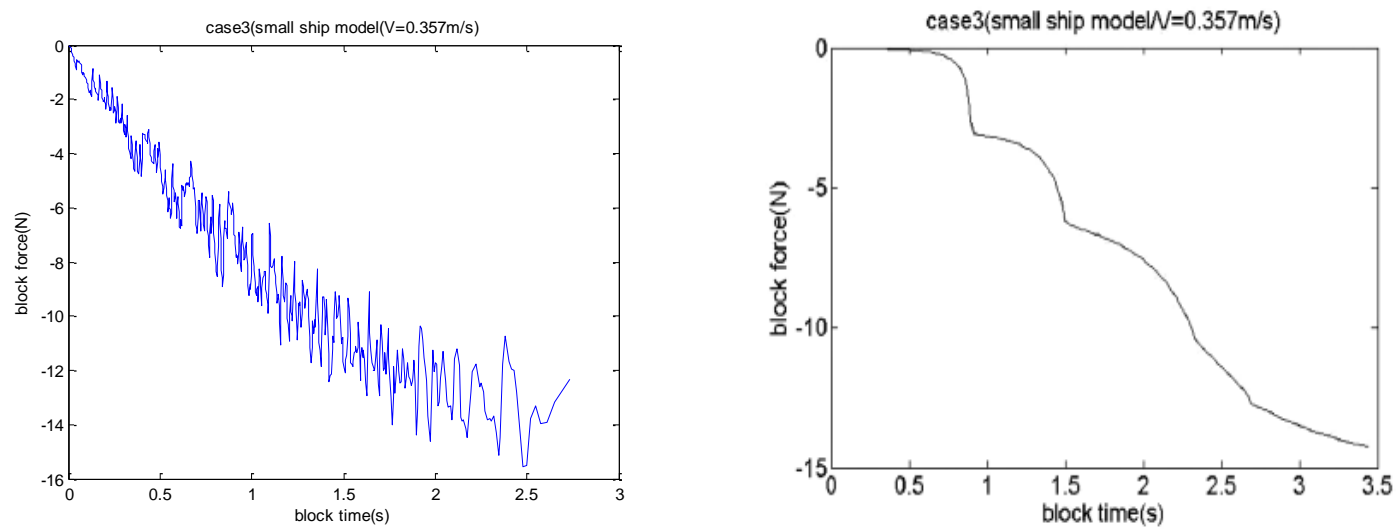

(c) case $3($ small model $\mathrm{V}=0.357 \mathrm{~m} / \mathrm{s})$ 

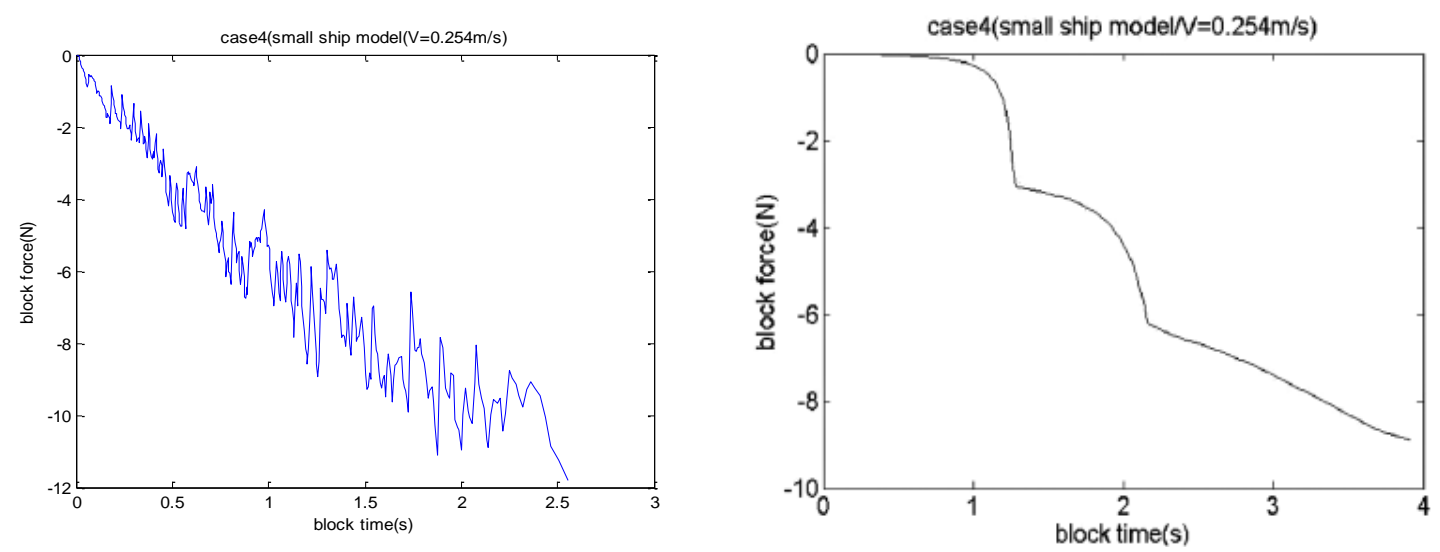

(d) case 4（ small model $\mathrm{V}=0.254 \mathrm{~m} / \mathrm{s}$ )

Figure 14 Time history curves of the block forces of 4 forward heading collision cases

\section{Conclusions}

In this paper, the forces of the various elements of the FFCPS were analyzed at first, and then based on a set of logical assumptions, the method of "position adjustment to approach equilibrium condition" was established. The feasibility and validation of this method have been verified, when considering different arrangements for the design of the FFCPS, by comparing with the measurements from model tests and published numerical predictions. Using the current numerical simulation method, the following can be concluded on the FFCPS:

(1) In the numerical calculation, a linear elastic model can be used to calculate the tensions of the connecting cable chains. The main factor of deformation is the mass and number of anchors.

(2) The block force is directly related to two factors. One is the pulling forces of the connecting cable chains, the other is the angles between the connecting cable chains and the ship's longitudinal axis. If the ship collides with the FFCPS at the same position, the order of the values of the anchor moving distances is certain. In ideal conditions, this order does not depend on the type and the speed of the colliding ship.

These conclusions are obtained under certain assumptions and can be used as a reference in engineering practice. Based on the calculation method, reasonably good advice can be obtained for the design, e.g. the number of anchors and their corresponding weights of the mooring system of a FFCPS. Furthermore, the type of the cable chains and their pre-tension could be ascertained. According to the maximum distance of the anchor movement, the safe distance between the FFCPS and the bridge can also be selected. However, in this paper, there is no consideration of some important effects, such as those from waves, wind and hydrodynamic effects. Dealing with such effects requires more in-depth studies, as well as more in-depth analysis of the experimental data. 


\section{References}

[1] Yun H, Nayeri R, Tasbihgoo F, et al. Monitoring the collision of a cargo ship with the Vincent Thomas Bridge[J]. Structural Control and Health Monitoring, 2008, 15(2): 183-206.

[2] Fan W, Yuan W C. Ship bow force-deformation curves for ship-impact demand of bridges considering effect of pile-cap depth[J]. Shock and Vibration, 2014: 1-19.

[3] Tam C K , Bucknall R. Collision risk assessment for ships[J]. Journal of Marine Science and Technology, 2010, 15(3): 257-270.

[4] Chen H T, Pan H J, Yao X Q. Collision-proof warning system for sea-cross bridge[J]. Advances in Intelligent and Soft Computing, 2012,133: 813-818.

[5] Gucma, L. Review of acceptable risk levels of bridge collapse in respect of ships collisions[J]. Safety and Reliability: Methodology and Applications-Proceedings of the European Safety and Reliability Conference, 2015:585-590.

[6] Mostefa M S. The branch-and-bound method and genetic algorithm in avoidance of ships collisions in fuzzy environment[J]. Polish Maritime Research, 2012, 19: 45-49.

[7] Hornauer S. Decentralised collision avoidance in a semi-collaborative multi-agent system[J]. Multiagent System Technologies Lecture Notes in Computer Science, 2013, 8076: 412-415.

[8] Agnieszka L. Safe ship control method with the use of ant colony optimization[J]. Solid State Phenomena, 2014, 210: 234-244.

[9] Kim, H J. Lim, J Y. Park, Wonsuk. Koh, Hyun-Moo1. Risk assessment of dolphin protected bridge pier considering collision point analysis[J]. Conference Proceedings of the Society for Experimental Mechanics Series, 2011, 4: 163-172.

[10] Obisesan A, Sriramula S, Harrigan J. Probabilistic considerations in the damage analysis of ship collisions[J]. Numerical Methods for Reliability and Safety Assessment, 2015: 197-214.

[11] Wang L L, Yang L M, Tang C G, Zhang Z W, Chen G Y, Lu Z L. On the impact force and energy transformation in ship-bridge collisions[J]. International Journal of Protective Structures, 2012, 3(1): 105-120.

[12] Wei L, Lu R L, Ning X L, Yuan Y, Hu Y X, Zeng H J. A mechanical calculation of the flexible \& floating anti-ship collision device for bridge piers[J]. Advanced Materials Research, 2012, 479-481: 2540-2545.

[13] Liu J, Yang Y. Structural optimization design of flexible anti-collision device for bridge[J]. Applied Mechanics and Materials, 2012, 201-202: 649-652.

[14] Lei Z B, Chen Z, Lei M X,Ai R. Research on safety technology for initiative anti-collision of bridge[J]. Applied Mechanics and Materials, 2012, 204-208:2196-2199.

[15] Zhu B, Chen R P, Chen Y M, Zhang Z H. Impact model tests and simplified analysis for flexible pile-supported protective structures withstanding vessel collisions[J]. Journal of Waterway, Port, Coastal 
and Ocean Engineering, 2012, 138(2): 86-96.

[16] Chang L H, Jiang C B, Liao M J, Xiao X. Nonlinear dynamic response of buoys under the collision load with ships[J]. Applied Mechanics and Materials, 2012, 204-208: 4455-4459.

[17] Wu G H, Yu Q L, Chen X J. An energy consumed collision-prevention system of long distance anchor moving for non-navigational bridge[J]. Highway, 2009, (1): 213-218. (in Chinese)

[18] Chen X J, Huang G Y, Wu G H, et al. Energy balance relationship in collision between ship and moored collision-prevention system[J]. Journal of PLA University of Science and Technology(Natural Science Edition), 2009, 10(1): 71-76. (in Chinese)

[19] Chen X J, Huang G Y, Wu G H, et al. New numerical method for flexible floating collision-prevention system and its convergency discussion[J]. Journal of PLA University of Science and Technology(Natural Science Edition), 2011, 12(5): 501-506. (in Chinese)

[20] Chen X J, Huang G Y, Wu G H, et al. Numerical simulation for the motion of the flexible floating collision-prevention system[J]. Journal of Offshore Mechanics and Arctic Engineering, 2013, 135:1-9.

[21] Xie Y H, Li Z Z. Construction Technology of Interception System for Ships on Non-navigable Openings of Hangzhou Bay Major Bridge [J]. Construction Technology, 2011, 40(3): 1-4.

[22] Yu Z, Shen Y, Amdahl J, et al. Implementation of linear potential-flow theory in the 6DOF coupled simulation of ship collision and grounding accidents[J]. Journal of Ship Research, 2016, 60(3):119-144

[23] JTS 144-1-2010, Load Code for Harbor Engineering[S], 2010. (in Chinese)

[24] Wei J D, Liu Z Y. Four sets of static solutions for elastic catenary[J]. Spatial Structures, 2005, 11(2):42-45.(in Chinese)

[25] Chen X J, Yu W, Liu J Y. The influence of cable chain connecting elastic modulus on the energy conversion of the Flexible Floating Collision-prevention system[J]. Journal of Traffic and Transportation Engineering, 2016, 16(3): 46-54. (in Chinese) 